\title{
La formulación en el nuevo curriculum de química
}

\author{
Juan Hernández y Laly Palacín
}
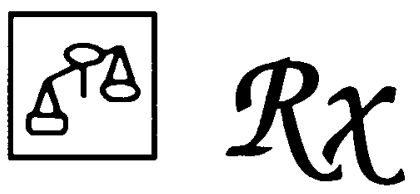

Sorprendentemente, cuando la formulación fue medio y producto de la comprensión en la bistoria de la química, boy su papel pedagógico no tiene nada que ver con esa función intelectual de ver y recordar. Actitudes negativas ante las ciencias y olvido de lo aprendido son reacciones esperables de los alumnos ante una enseñanza no signifiativa de la formulación. Partiendo de estos becbos, los autores proponen dotar de significatividad a la formulación, impartiéndola a partir de los elementos químicos formulados $y$ graduando su enseñanza de acuerdo con ellos.

\section{INTRODUCCION}

"Cuando yo era jóven e indocumentado, una de las muchas tareas que me agobiaban era el estudio de largas e incomprensibles formulaciones, llenas de letras grandes y pequeños números que a veces se fundían para producir otras fórmulas aún más raras y difíciles Las letras eran símbolos que representaban elementos de una tabla inacabable que había que memorizar periódicamente. Según decían, estaba aprendiendo Química. Pero sólo hoy, unos cuantos años después, de aquellos conocimientos químicos sólo quedan algunos vestigios, piezas de un puzzle tal vez nunca acabado. perdidas en algún rincón de la memoria donde reposan también los Reyes Godos y alguna que otra batalla célebre". Este párrafo correspondiente a J 1. Pozo en una conferencia celebrada dentro de los IV Encuentros sobre aspectos didácticos en las 


\section{2}

EEMM (Pozo, 1988), describe de forma real y objetiva la situación de un elevado porcentaje de estudiantes que deciden no elegir la Química al finalizar el actual 2 de BUP.

En realidad la similitud entre química y tormulación es algo que nosotros mismos hemos podido detectar al entrevistar a diversos alumnos y alumnas de 1 y 2 de BUP. Muchos eran los que al preguntarles lo que creían que era la química solían responder en términos similares a: "La química...Ah! eso de las tórmulas".

Esta situación es coherente con la importancia que una buena parte del profesorado de EGB da a la formulación dentro del curriculum de química $\mathrm{y}$ de hecho responde también al sentido de una parte del protesorado de BUP que suele utilizar los resultados que muestran lo poco que recuerdan de esta parte para indicar "el bajo nivel del alumnado en química"

En la cita de Pozo, el autor señala también: "Hoy, unos cuantos años despues, las cosas han cambiado bastante. En los libros y en las aulas de química, como en otras materias escolares se busca cada vez más la comprensión. La química ya no sólo se memoriza; también se comprende."

Sin embargo, nosotros creemos que esta afirmación no es general. Sí qué existe una clara tendencia a hacer realidad la afirmación de Pozo y de hecho, año tras añio, se produce un aumento del número de profesores y profesoras que deciden sustituir la ensenanza de la formulación por otros temas con un contenido formativo más interesante. Pero todavía es mayoritario el profesorado de ciencias de secundaria que la considera parte básica del curriculum de química y que establece pruebas específicas de evaluación para obligar al alumnado a memorizar nombres y fórmulas de sustancias sobre las que no han oído hablar nada de ellas, e incluso se les propone en muchas ocasiones otras que ni siquiera existen.

En consecuencia, dado que estamos actualmente inmersos en un proceso que conducirá a la elaboración de los proyectos curriculares de área en la etapa secundaria y, posteriormente, en el Bachillerato, consideramos importante analizar cuando es conveniente y pertinente introducir la formulación como uno de los contenidos del curriculum.

\section{LA FORMULACION EN EL CURRICULUM ACTUAL}

Para conocer la situación del proceso de enseñanza-aprendizaje en el que se encuentra la parte correspondiente a la nomenclatura y formulación de los compuestos químicos, hemos comenzado por acudir a un estudio de libros de texto dado que la investigación didáctica señala que este método permite conocer, de forma aproximada el curriculum desarrollado por un alto porcentaje de profesores y profesoras así como su concreción en el aula.

El análisis lo hemos hecho sobre 14 textos de $8^{\circ}$ de EGB y 12 de $2^{\circ}$ de BUP. De entre ellos el $82 \%$ de los textos de EGB consultados y el $75 \%$ de 
los de BUP incluyen de forma expícita apartados dedicados a la formulación inorgánica. La mayoría lo realiza formulando todo tipo de compuesto químico sin importar si existe o no. Y así no es raro encontrar nombres similares al hidrocarbonato de cobalto 11 o el inexistente ácido carbónico. Por supuesto, en ninguno de los libros consultados se comenta nada de la posible utilidad de algunos de ellos, aunque sean habituales, ni se les informa sobre el nombre con el que algunos de ellos se le conoce habitualmente y que permitiría relacionarlos con sustancias de uso común (por ejemplo, el mármol, el salffumán, la sosa, etc.); y ello, a pesar de que muchos de ellos suelen venir con tres nombres distintos (sistemática, de Stock y tradicional). El tema se presenta como un rosario de normas o reglas, como si de una receta se tratase, donde, si se van cumpliendo los pasos al final, el resultado sale bien.

Esta situación de los libros de texto es suficientemente indicativa de la importancia que al tema da una mayoría del profesorado. Nosotros mismos hemos podido comprobarlo personalmente a través de los numerosos cursos de formación a los que hemos asistido y en los que hemos detectado la preocupación que por el tema muestra el profesorado (los de BUP por la dificultad que encuentran en lograr que sus alumnos dominen esta parte, y los de EGB por la acusación que sufren por algunos compañeros de niveles superiores referentes a lo gue según éstos es demostrativo de un "bajo nivel de conocimientos de química con los que proceden"), también hemos detectado el rechazo inicial sobre nuestra propuesta basada en dudar de la imprescindibilidad que la formulación tiene en el curriculum de la químcia de secundaria.

Así pues, puede afirmarse que una mayoría del profesorado que imparte Química a partir de $8^{\circ}$ de EGB dedican una parte del tiempo destinado a esta materia a la enseñanza de la nomenclatura y formulación de los compuestos químicos. $Y$ sin embargo, a pesar del tiempo dedicado a la enseñanza, el profesorado de EEMM es conocedor de las dificultades que el alumnado, no sólo de $2^{\circ}$, sino también de 30 y COU, tiene. De hecho los Seminarios didácticos, conscientes de la situación, establecen procedimientos para tratar de que los alumnos que eligen Química lleguen a aprender a formular con la suficiente desenvoltura.

Es decir, que nos encontramnos ante lo que es una clara muestra de un aprendizaje poco significativo. Un caso anecdótico pero que refleja el pensamiento generalizado del profesorado de EGB que imparte Química, fue el que sucedió en el CEP de Valencia en un curso de formación dirigido al profesorado de varios centros de una misma zona. En dicho curso defendimos la propuesta de no incluir la formulación en este nivel dada, entre otros factores, la poca significatividad de los mismos. Esta propuesta no era, en general, bien aceptada, pues todos los asistentes, a excepción de uno de ellos, dedicaban una parte de la enseñanza de la química a ella. Por esta razón propusimos analizar los resultados de un trabajo consistentes en comprobar la formulación que los alumnos recordaban antes de comenzar la química en BUP, para lo que se disponían de las respuestas dadas por 178 alumnos de $1^{\circ}$ y $2^{\circ}$. En este trabajo se comprobaba que si bien el $98 \%$ supieron escribir la 


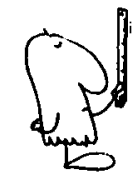

fórmula correcta del agua, sólo el 17\% supo la del cloruro de sodio, el $11 \%$ la del óxido de calcio, el 6,8\% la del amoníaco, el 1,3\% la del ácido clorhídrico y el $0,6 \%$ de le los ácidos nítrico y sulfúrico, entre otros resultados.

Al analizar los resultados los asistentes aducían a factores ligados con la metodología o el escaso tiempo que el profesorado presumiblemente habría dedicado a su enseñanza; sin embargo, cuando se les comunicó que esos datos habían sido extraidos esa misma semana del instituto al que asistían los que habían sido sus alumnos mostraron una gran sorpresa al reconocer entre los encuestados alumnos que habían sido capaces, hacía uno o dos años, de superar con éxito las pruebas diseñadas para comprobar su aprendizaje.

Ahora bien, sería totalmente injusto considerar que el problema de la falta de un aprendizaje significativo de la formulación sólo se produce en EGB pues un estudio de la Universidad Politécnica de Valencia dirigido a conocer cual es la química que saben los estudiantes cuando acceden a esta Universidad detectó que el $43 \%$ de los presentes no sabían formular correctamente el sulfato de sodio, el $36 \%$ el cloruro de calcio y el 31\% el hidróxido de hierro III. Como puede apreciarse estos datos correpondientes a estudiantes que la mayoria ha estado formulando los tres últimos años son un claro exponente de un problema relacionado con el aprendizaje.

\section{LA QUMICA Y LA FORMULACION}

Es decir que nos encontramos ante un tema considerado importante por parte del profesorado y con un claro exponente de una falta de aprendizaje significativo. Vamos en primer lugar a analizar las posibles causas que justifiquen esa falta de signiticatividad.

Para Ausubel (1978) los conocimientos de una persona están organizados en una estructura cognitiva formada por conceptos y sus relaciones y un concepto tiene significado para un individuo si llega a formar parte de su estructura cognitiva de una manera intencionada. Por otra parte, como señala Coll (1987) para lograr aprendizajes significativos debe existir una disposición favorable por parte del alumnado, de ahí la necesidad de lograr actitudes positivas hacia los temas enseñados y como señala Polya (1980) la respuesta de los estudiantes a su enseñanza depende de sus antecedentes, sus perspectivas y sus intereses. Aplicando estas ideas al tema de la formulación podemos encontrar argumentos para justificar los resultados analizados anteriormente.

Ahora bien el problema que tenemos planteado es el de decidir si la formulación de productos químicos debe formar parte del curriculum, $y$ una vez decidido el cuanto deberemos entrar en buscar la metodología adecuada para que el aprendizaje sea significativo.

Para seleccionar contenidos que puedan ser aprendidos significativamente resulta de gran importancia conocer aquello que a los estu- 
diantes les gustaría saber y lo que no tienen interés en saber así como lo que nosotros, como expertos, consideramos lo que debe ser imprescindible que aprendan y aquello que no es $\tan$ importante que sepan (Polya).

Respecto a la primera condición, el tema de la formulación difícilmente puede ser un tema que provoque interés para el alumnado; pues, el conocimiento de las propiedades y aplicaciones de un producto químico no es asociado, en estas edades, con la fórmula. Asi, los efectos del salfumán sobre el mármol pueden conocerse perfectamente sin conocer la fórmula de estos compuestos.

En consecuencia la elección va a depender del interés que, nosotros, como profesores consideremos que esta parte tiene.

Es evidente que los contenidos seleccionados deben servir para dar una visión correcta de la materia teniendo en cuenta la edad a los que van dirigidos. Obviamente un primer dato a considerar lo constituye los objetivos que se pretende cumplir. Como objetivo general básico consideramos el contribuir a formar individuos con capacidades de pensar por sí mismos ayudándoles a potenciar al máximo sus capacidades favoreciendo la familiarización con el método científico y como objetivo más general de la Química el ayudar a la comprensión de la Naturaleza a partir de la estructura de la materia y de su transformación sin olvidar el de contribuir a lograr una visión crítica de la ciencia y de la utilización que de ella se hace.

Un dato que merece consideración lo constituyen los estudios de Schibeci (1984) en los que se demuestra el progresivo desinterés que muestran los estudiantes conforme aumenta sus estudios en estas materias; 0 , los que hablan de la huida de las clases de ciencias (Matthews, 1991) o los más recientes que tratan el tema de las actitudes frente a las ciencias (Solbes y Vilches, 1988).

En consecuencia, después de todo lo expuesto opinamos que esta parte de la Química no debe formar parte del curriculum de una etapa obligatoria como es la Educación Secundaria (12-16) al considerar que por una parte el conocimiento de la fórmula no es necesario para comprender los procesos químicos que se estudia en ese nivel $y$, por otra, que el estudio de esta parte no contribuye a cumplir los objetivos señalados, contrariamente a otros temas que si son válidos para lograrlo.

Ahora bien, en la enseñanza postobligatoria (etapa 16-18) la situación es distinta pues consideramos que los estudiantes que acceden a estudios universitarios en los que van a utilizar la química, el dominio de la nomenclatura y formulación es importante para afrontar con éxito el estudio de esta materia.

Un aspecto que debe ser comentado es considerar que la no exigencia del dominio de la formulación es independiente del estudio de la escritura de reacciones o de la realización de ejercicios de estequiometría pues lo único que supone es que al alumno se le facilita la fórmula correcta de los compuestos que intervienen. 


\section{LA FORMULACION EN EL BACHILLERATO}

Por tanto una vez decidido que la formulación forma parte del curriculum del bachillerato procede ofrecer una propuesta sobre su enseñanza que permita un aprendizaje signiticativo y conseguir, así, que los estudiantes que han optado por la química finalicen los estudios no universitarios con un dominio básico sobre la nomenclatura y formulación de compuestos químicos.

Comenzamos por considerar que la formulación, como cualquier otro lenguaje simbólico, surge frente a la necesidad de representar algo y para que esta función sea racional y por tanto humana parece evidente que habrá que poseer algún conocimiento acerca de aquello que pretendemos representar (LLorens, 1991). Por lo que somos partidarios de acompañar la ensenanza de la formulación con información sobre aquellos compuestos que por su utilización o abundancia pueden resultarles interesantes para acercarles el estudio de la química al entorno. La mayoría de los alumnos de COU son capaces de formular correctamente compuestos como el carbonato de calcio, hidróxido sódico u óxido de calcio sin relacionarlos con ninguno de los productos de uso cotidiano (Cerdán et al, 19)

Por otra parte, rechazamos la ensenanza de la nomenclatura y formulación de productos químicos como únicamente un rosario de normas y reglas sin aparente justificación. Consideramos que si bien es necesario dar reglas éstas pueden y deben ir acompañadas de alguna justificación que permita comprender la fórmula. Ello supone graduar su ensenanza de forma que la formulación de compuestos binarios se aborde en el tema correspondiente al enlace químico mientras que los corespondientes a las sales procedentes de ácidos ternarios sean estudiados en el momento en que se trata el tema correspondiente a reacciones de neutralización procedentes de ácidos e hidróxidos.

Consideramos que el hecho de exigir el conocimiento de las fórmulas correctas de productos que ha venido utilizando y con los que presumiblemente se encuentran ya familiarizados facilita su aprendizaje.

Por último queremos también señalar nuestra oposición a formular multitud de compuestos aconsejando evitar los que no existen y centrarnos principalmente a los más comunes.

\section{Referencias}

Atsuber, D. P.(1978): Psicologia educativa: un punto de vista cognitivo. México: Trillas.

CERDAN et al.(1985): Disociación entre la quimica escolar y la realidad. I Congreso Internacional sobre investigación en la didáctica de las Ciencias y de las Matemáticas. Barcelona.

Cou C. (1987). Psicología y curriculum. Barcelona: Laia.

LLORENS J. A. (1991) Comenzando a aprender química. Madrid: Visor.

MATTEHEws M. (1991), Un lugar para la historia y la filosofía en la enseñanza delas ciencias. CLEE. $(11-12) 141-155$.

PoLy^ G. (1980) On solving mathematical problems in high school. In S. Krulik and R.E. Key editurs. ProbleM SOLWING IN SCHOOL MathEMATICS. VirGini.

Pozo, J.L. (1988) Procesos psicológicos en el cambio conceptual en Química. Aspectos didácticos de Física y Química ll-36. ICE. Universidad de Zaragoza.

Schibec1, R. A. (1984) Attitudes to science: an update. Srudies in Science Education, 70 (2), 1391 49.

SOLBES J. Y VILChes, A. (1989). Interacciones $\mathrm{C} / \mathrm{T} / \mathrm{S}$ : un instrumento de cambio actitudinal. Enseñanza de las Ciencias 7, (1) 14-20. 
La formulación en el nuevo curriculum de química

\author{
Juan Hernández Pérez y Laly Palacín Fernández \\ CL\&E, 1993, 19-20, 101-107
}

Resumen: Creemos necesario reformular el tratamiento que al estudio de la Química se le está dando, en algunos casos, en la etapa obligatoria 12-16, ya que son todavía muchos los profesores que dedican un período de tiempo más o menos largo al estudio de la formulación química. Este estudio se hace de una forma memorística donde el único objetivo es que el alumno aprenda una serie de fórmulas, para superar una prueba escrita, pero sin profundizar en el significado de las expresione o su posible aplicación en la vida real. $Y$ dado que para la comprensión de la Química en esta etapa obligatoria no se considera necesario que el alumno sepa formular o no, pensamos que sería más conveniente dejarlo para la enseñanza postobligatoria (16-18), ya que los estudiantes que quieran acceder a estudios universitarios en los que van a utilizar la química, el dominio de la nomenclatura y formulación es importante para afrontar con éxito el estudio de esta materia. Ahora bien, no es todo decidir cuándo se va a tratar este tema sino cómo se va a hacer para que permita un aprendizaje significativo, de manera que cuando los alumnos se encuentren cursando sus estudios superiores no tengan que enfrentarse de nuevo al estudio de ciertos compuestos básicos ya que ellos mismos reconocen que no los recuerdan, tal y como hemos comprobado en los items pasados a estudiantes universitarios de carreras de ciencias.

Datos sobre los autores: Juan hernández es licenciado en Ciencias Químicas y actualmente es director del CEP de Valencia. Laly Palacin es Licenciada en Ciencias biológicas y actualmente es profesora de $2^{\mathbf{a}}$ etapa de EGB en la asignatura Ciencias Naturales.

Dirección: CEP Valencia, C\&/Juan de Garay, 25, 46017 Valencia

(1) De todos los artículos deberá solicitarse por escrito autorización de CL\&E y de los autores para el uso en forma de facsímil, fotocopia o cualquier otro medio de reproducción impresa. CL\&E se reserva el derecho de interponer acciones legales necesarias en aquellos casos en que se contravenga la ley de derechos de autor. 